\title{
Some results concerning localization property of generalized Herz, Herz-type Besov spaces and Herz-type Triebel-Lizorkin spaces
}

\author{
Djeriou A., Heraiz R.
}

In this paper, based on generalized Herz-type function spaces $\dot{K}_{q}^{p}(\theta)$ were introduced by Y. Komori and K. Matsuoka in 2009, we define Herz-type Besov spaces $\dot{K}_{q}^{p} B_{\beta}^{s}(\theta)$ and Herz-type Triebel-Lizorkin spaces $\dot{K}_{q}^{p} F_{\beta}^{s}(\theta)$, which cover the Besov spaces and the Triebel-Lizorkin spaces in the homogeneous case, where $\theta=\{\theta(k)\}_{k \in \mathbb{Z}}$ is a sequence of non-negative numbers such that

$$
C^{-1} 2^{\delta(k-j)} \leq \frac{\theta(k)}{\theta(j)} \leq C 2^{\alpha(k-j)}, \quad k>j,
$$

for some $C \geq 1$ ( $\alpha$ and $\delta$ are numbers in $\mathbb{R}$ ).

Further, under the condition mentioned above on $\theta$, we prove that $\dot{K}_{q}^{p}(\theta)$ and $\dot{K}_{q}^{p} B_{\beta}^{s}(\theta)$ are localizable in the $\ell_{q}$-norm for $p=q$, and $\dot{K}_{q}^{p} F_{\beta}^{s}(\theta)$ is localizable in the $\ell_{q}$-norm, i.e. there exists $\varphi \in \mathcal{D}\left(\mathbb{R}^{n}\right)$ satisfying $\sum_{k \in \mathbb{Z}^{n}} \varphi(x-k)=1$, for any $x \in \mathbb{R}^{n}$, such that

$$
\|f \mid E\| \approx\left(\sum_{k \in \mathbb{Z}^{n}}\|\varphi(\cdot-k) \cdot f \mid E\|^{q}\right)^{1 / q}
$$

Results presented in this paper improve and generalize some known corresponding results in some function spaces.

Key words and phrases: generalized Herz space, Herz-type Besov space, Herz-type TriebelLizorkin space, localization property.

Laboratory of Mathematics Pure and Applied, M'Sila University, P.O. Box 166, M'Sila 28000, Algeria

E-mail: aissa.djeriou@univ-msila.dz(Djeriou A.), rabah.heraiz@univ-msila.dz (Heraiz R.)

\section{Introduction and preliminaries}

As usual, $\mathbb{R}^{n}$ is the $n$-dimensional real Euclidean space, $\mathbb{N}$ is the collection of all natural numbers and $\mathbb{N}_{0}=\mathbb{N} \cup\{0\}$. The letter $\mathbb{Z}$ stands for the set of all integer numbers.

For any $u>0, k \in \mathbb{Z}$ we set $C(u)=\left\{x \in \mathbb{R}^{n}: u / 2<|x| \leq u\right\}$ and $C_{k}=C\left(2^{k}\right)$. For $x \in \mathbb{R}^{n}$ and $r>0$ we denote by $B(x, r)$ the open ball in $\mathbb{R}^{n}$ with center $x$ and radius $r$. Let $\chi_{k}$, for $k \in \mathbb{Z}$, denote the characteristic function of the set $C_{k}$.

As usual, $L^{p}\left(\mathbb{R}^{n}\right)$ for $0<p \leq \infty$ stands for the Lebesgue spaces on $\mathbb{R}^{n}$ normed by (quasinormed for $p<1$ )

$$
\left\|f \mid L^{p}\left(\mathbb{R}^{n}\right)\right\|=\|f\|_{p}=\left(\int_{\mathbb{R}^{n}}|f(x)|^{p} d x\right)^{1 / p}<\infty, \quad 0<p<\infty,
$$

$\mathrm{y} \Delta \mathrm{K} 517.98$

2010 Mathematics Subject Classification: 46E30, 46E35, 42B35. 
and

$$
\left\|f\left|L^{\infty}\left(\mathbb{R}^{n}\right)\|=\| f \|_{\infty}=\underset{x \in \mathbb{R}^{n}}{\operatorname{ess}}\right| f(x) \mid<\infty .\right.
$$

By $\ell_{q}, 0<q \leq \infty$, we denote the space of all (complex) sequences $\left\{a_{k}\right\}_{k \in \mathbb{Z}}$ equipped with the quasi-norm

$$
\left\|\left\{a_{k}\right\}_{k \in \mathbb{Z}} \mid \ell_{q}\right\|=\left(\sum_{k=-\infty}^{\infty}\left|a_{k}\right|^{q}\right)^{1 / q}
$$

(with the usual modification if $q=\infty$ ).

Given two quasi-Banach spaces $X$ and $Y$, we write $X \hookrightarrow Y$ if $X \subset Y$ and the natural embedding of $X$ in $Y$ is continuous. We use $c$ as a generic positive constant, i.e. a constant whose value may change from appearance to appearance.

By $\mathcal{S}\left(\mathbb{R}^{n}\right)$ we denote the Schwartz space of all complex-valued, infinitely differentiable and rapidly decreasing functions on $\mathbb{R}^{n}$. The topology in the complete locally convex space $\mathcal{S}\left(\mathbb{R}^{n}\right)$ is generated by the norms

$$
p_{N}(\varphi)=\sup _{x \in \mathbb{R}^{n}}(1+|x|)^{N} \sum_{|\alpha| \leq N}\left|D^{\alpha} \varphi(x)\right|, \quad N=1,2,3, \ldots
$$

By $\mathcal{S}^{\prime}\left(\mathbb{R}^{n}\right)$ we denote the dual space of all tempered distributions on $\mathbb{R}^{n}$. We define the Fourier transform of a function $f \in \mathcal{S}\left(\mathbb{R}^{n}\right)$ by

$$
\mathcal{F}(f)(\xi)=(2 \pi)^{-n / 2} \int_{\mathbb{R}^{n}} e^{-i x \cdot \xi} f(x) d x .
$$

Its inverse is denoted by $\mathcal{F}^{-1} f$. Both $\mathcal{F}$ and $\mathcal{F}^{-1}$ are extended to the dual Schwartz space $\mathcal{S}^{\prime}\left(\mathbb{R}^{n}\right)$ in the usual way.

It is well known that the localization property was first introduced by G. Bourdaud (see [1]) and under some conditions he proved that Besov spaces is localizable in the $\ell_{p}$ norm. N. Ferahtia and S. Allaoui (see [4]) generalized the Bourdaud theorem of a localization property of Besov spaces $B_{p, q}^{s}$ on the $\ell_{r}$ space, where $r \in[1,+\infty]$.

Recently, the localization property of some function spaces have attracted great attention (see $[11,13,20])$.

In this paper, we define Herz-type Besov spaces $\dot{K}_{q}^{p} B_{\beta}^{s}(\theta)$ and Herz-type Triebel-Lizorkin spaces $\dot{K}_{q}^{p} F_{\beta}^{s}(\theta)$ which covers Besov spaces and Triebel-Lizorkin spaces in the homogeneous case. Notice that these spaces based on generalized Herz-type function spaces $\dot{K}_{q}^{p}(\theta)$ were introduced by Y. Komori and K. Matsuoka in [8]. After this, we treat and discuss the localization property of these spaces and then we compare our results with existing ones.

\section{Function spaces}

We start by recalling the definition and some properties of the generalized Herz spaces.

Definition 1. Let $\alpha, \delta \in \mathbb{R}$. A sequence of numbers $\theta=\{\theta(k)\}_{k \in \mathbb{Z}}$ belongs to the class $\mathcal{A}(\alpha, \delta)$ if and only if

(i) $\theta(k)>0$ for all $k \in \mathbb{Z}$;

(ii) there exists a constant $C \geq 1$ such that

$$
C^{-1} 2^{\delta(k-j)} \leq \frac{\theta(k)}{\theta(j)} \leq C 2^{\alpha(k-j)}
$$

for $k>j$. 
The size condition (1) in the above definition can be satisfied by many sequences of numbers such as:

$$
\theta=\left\{2^{\mu k}\right\}_{k \in \mathbb{Z}} \in \mathcal{A}(\alpha, \delta) \text { for } \delta \leq \mu \leq \alpha, \quad \theta=\left\{2^{\lambda k}(1+\max (0, k \ln 2))\right\}_{k \in \mathbb{Z}} \in \mathcal{A}(\lambda, \lambda+1) .
$$

Definition 2. Let $\theta \in \mathcal{A}(\alpha, \delta)$ and $0<p, q \leq \infty$. The generalized Herz space $\dot{K}_{q}^{p}(\theta)$ is defined by

$$
\dot{K}_{q}^{p}(\theta):=\left\{f \in L_{\mathrm{loc}}^{q}\left(\mathbb{R}^{n} \backslash\{0\}\right):\left\|f \mid \dot{K}_{q}^{p}(\theta)\right\|<\infty\right\},
$$

where

$$
\left\|f \mid \dot{K}_{q}^{p}(\theta)\right\|=\left(\sum_{k=-\infty}^{\infty} \theta^{p}(k)\left\|f \chi_{k} \mid L^{q}\right\|^{p}\right)^{1 / p},
$$

with the usual modifications made when $p=\infty$ and/or $q=\infty$.

The spaces $\dot{K}_{p}^{q}(\theta)$ were first defined by Y. Komori and K. Matsuoka [8] and under the condition above, the authors studied the boundedness of singular integral operators and fractional integral operators on these spaces.

The Definition 2 coincide with the classical definition of Herz spaces for the case of the particular function, i.e

$$
\dot{K}_{q}^{p}(\theta)=\dot{K}_{q}^{\alpha, p}\left(\mathbb{R}^{n}\right) \quad \text { if } \quad \theta \in \mathcal{A}(\alpha, \alpha) .
$$

The spaces $\dot{K}_{q}^{p}(\theta)$ are quasi-Banach spaces and if $\min (p, q) \geq 1$ then $\dot{K}_{q}^{p}(\theta)$ are Banach spaces. If $\theta \in \mathcal{A}(0,0)$ and $0<p=q \leq \infty$ then $\dot{K}_{p}^{p}(\theta)$ coincides with the Lebesque spaces $L^{p}\left(\mathbb{R}^{n}\right)$. A detailed discussion of the properties of $\dot{K}_{q}^{p}(\theta)$, where $\theta \in \mathcal{A}(\alpha, \alpha)$, may be found in the papers $[6,7,9,10]$, and references therein.

Next, we present the Fourier analytical definition of Herz-type Besov spaces $\dot{K}_{q}^{p} B_{\beta}^{s}(\theta)$ and Herz-type Triebel-Lizorkin spaces $\dot{K}_{q}^{p} F_{\beta}^{s}(\theta)$ and recall their basic properties. We first need the concept of a smooth dyadic resolution of unity.

Definition 3. Let $\Psi$ be a function in $\mathcal{S}\left(\mathbb{R}^{n}\right)$ satisfying $\Psi(x)=1$ for $|x| \leq 1$ and $\Psi(x)=0$ for $|x| \geq \frac{3}{2}$. We put $\varphi_{0}(x)=\Psi(x), \varphi_{1}(x)=\Psi(x / 2)-\Psi(x)$ and

$$
\varphi_{j}(x)=\varphi_{1}\left(2^{-j+1} x\right) \quad \text { for } j=2,3, \ldots
$$

Then we have supp $\varphi_{j} \subset\left\{x \in \mathbb{R}^{n}: 2^{j-1} \leq|x| \leq 3 \cdot 2^{j-1}\right\}, \varphi_{j}(x)=1$ for $3 \cdot 2^{j-2} \leq|x| \leq 2^{j}$ and $\sum_{j=0}^{\infty} \varphi_{j}(x)=1$ for all $x \in \mathbb{R}^{n}$. The system of functions $\left\{\varphi_{j}\right\}_{j \in \mathbb{N}_{0}}$ is called a smooth dyadic resolution of unity. We define the convolution operators $\Delta_{j}$ as follows

$$
\Delta_{j} f=\mathcal{F}^{-1} \varphi_{j} * f, \quad j \in \mathbb{N}, \quad \text { and } \quad \Delta_{0} f=\mathcal{F}^{-1} \Psi * f, \quad f \in \mathcal{S}^{\prime}\left(\mathbb{R}^{n}\right) .
$$

Thus we obtain the Littlewood-Paley decomposition $f=\sum_{j=0}^{\infty} \Delta_{j} f$ of all $f \in \mathcal{S}^{\prime}\left(\mathbb{R}^{n}\right)$ (convergence in $\mathcal{S}^{\prime}\left(\mathbb{R}^{n}\right)$ ).

We are now ready to state the definitions of Herz-type Besov and Triebel-Lizorkin spaces.

Definition 4. (i) Let $\theta \in \mathcal{A}(\alpha, \delta), s \in \mathbb{R}$, and $0<p, q, \beta \leq \infty$. The generalized Herz-type Besov space $\dot{K}_{q}^{p} B_{\beta}^{s}(\theta)$ is the collection of all $f \in \mathcal{S}^{\prime}\left(\mathbb{R}^{n}\right)$ such that

$$
\left\|f \mid \dot{K}_{q}^{p} B_{\beta}^{s}(\theta)\right\|=\left(\sum_{j=0}^{\infty} 2^{j s \beta}\left\|\Delta_{j} f \mid \dot{K}_{q}^{p}(\theta)\right\|^{\beta}\right)^{1 / \beta}<\infty,
$$


with the obvious modification if $\beta=\infty$.

(ii) Let $\theta \in \mathcal{A}(\alpha, \delta), s \in \mathbb{R}, 0<p, q<\infty$ and $0<\beta \leq \infty$. The generalized Herz-type Triebel-Lizorkin space $\dot{K}_{q}^{p} F_{\beta}^{s}(\theta)$ is the collection of all $f \in \mathcal{S}^{\prime}\left(\mathbb{R}^{n}\right)$ such that

$$
\left\|f\left|\dot{K}_{q}^{p} F_{\beta}^{s}(\theta)\|=\|\left(\sum_{j=0}^{\infty} 2^{j s \beta}\left|\Delta_{j} f\right|^{\beta}\right)^{1 / \beta}\right| \dot{K}_{q}^{p}(\theta)\right\|<\infty,
$$

with the obvious modification if $\beta=\infty$.

Observing that, if $\theta \in \mathcal{A}(\alpha, \alpha)$ then $\dot{K}_{q}^{p} B_{\beta}^{s}(\theta)=\dot{K}_{q}^{\alpha, p} B_{\beta}^{s}\left(\mathbb{R}^{n}\right)$ (resp., $\dot{K}_{q}^{p} F_{\beta}^{s}(\theta)=\dot{K}_{q}^{\alpha, p} F_{\beta}^{s}$ ) are the classical Herz-type Besov spaces (resp., the classical Herz-type Triebel-Lizorkin spaces). The spaces $\dot{K}_{q}^{p} B_{\beta}^{s}(\theta)$ and $\dot{K}_{q}^{p} F_{\beta}^{s}(\theta)$ are quasi-Banach spaces and if $p, q, \beta \geq 1$, then both $\dot{K}_{q}^{p} B_{\beta}^{s}(\theta)$ and $\dot{K}_{q}^{p} F_{\beta}^{s}(\theta)$ are Banach spaces. Further results, concerning, for instance, lifting properties, Fourier multiplier and local means characterizations can be found in [17-19].

Now we give the definitions of the spaces $B_{p, \beta}^{s}$ and $F_{p, \beta}^{s}$.

Definition 5. (i) Let $s \in \mathbb{R}$ and $0<p, \beta \leq \infty$. The Besov space $B_{p, \beta}^{s}\left(\mathbb{R}^{n}\right)$ is the collection of all $f \in \mathcal{S}^{\prime}\left(\mathbb{R}^{n}\right)$ such that

$$
\left\|f \mid B_{p, \beta}^{s}\left(\mathbb{R}^{n}\right)\right\|=\left(\sum_{j=0}^{\infty} 2^{j s \beta}\left\|\Delta_{j} f \mid L^{p}\left(\mathbb{R}^{n}\right)\right\|^{\beta}\right)^{1 / \beta}<\infty .
$$

(ii) Let $s \in \mathbb{R}, 0<p<\infty$ and $0<\beta \leq \infty$. The Triebel-Lizorkin space $F_{p, \beta}^{s}\left(\mathbb{R}^{n}\right)$ is the collection of all $f \in \mathcal{S}^{\prime}\left(\mathbb{R}^{n}\right)$ such that

$$
\left\|f\left|F_{p, \beta}^{s}\left(\mathbb{R}^{n}\right)\|=\|\left(\sum_{j=0}^{\infty} 2^{j s \beta}\left|\Delta_{j} f\right|^{\beta}\right)^{1 / \beta}\right| L^{p}\left(\mathbb{R}^{n}\right)\right\|<\infty .
$$

The theory of the spaces $B_{p, q}^{s}\left(\mathbb{R}^{n}\right)$ and $F_{p, \beta}^{s}\left(\mathbb{R}^{n}\right)$ has been developed in detail in [14-16], but has a longer history already including many contributors; we do not want to discuss this here. In particular, with $p=q=\infty, s>0$, one recovers Hölder-Zygmund spaces $\mathcal{C}^{s}=B_{\infty, \infty}^{s}$ cf. [14, Theorem 2.5.12]. Clearly, for $\theta \in \mathcal{A}(0,0), s \in \mathbb{R}, 0<p \leq \infty\left(0<p<\infty\right.$ for the $\dot{K}_{p}^{p} F_{\beta}^{s}(\theta)$ spaces) and $0<\beta \leq \infty$,

$$
\dot{K}_{p}^{p} B_{\beta}^{s}(\theta)=B_{p, \beta}^{s}\left(\mathbb{R}^{n}\right) \quad \text { and } \quad \dot{K}_{p}^{p} F_{\beta}^{s}(\theta)=F_{p, \beta}^{s}\left(\mathbb{R}^{n}\right) .
$$

For the proof of the localization property of Herz-type Besov and Herz-type Triebel-Lizorkin spaces, we need the following proposition, see [2, Proposition 3.5].

Proposition 1. Let $\theta \in \mathcal{A}(\alpha, \delta), s \in \mathbb{R}$ and $1 \leq p, q, \beta \leq \infty$ such that $-n / q<\alpha, \delta<n(1-1 / q)$. For all $\gamma, \rho>1$, there exists $c>0$ such that for any sequence $\left\{g_{l}\right\}_{l \in \mathbb{N}_{0}}$ of functions, where

$$
\operatorname{supp} \mathcal{F} g_{0} \subset\{\xi:|\xi| \leq \rho\} \quad \text { and } \quad \operatorname{supp} \mathcal{F} g_{l} \subset\left\{\xi: \gamma^{-1} 2^{l} \leq|\xi| \leq \gamma 2^{l}\right\},
$$

we have

$$
\left\|\sum_{l=0}^{\infty} g_{l} \mid \dot{K}_{q}^{p} B_{\beta}^{s}(\theta)\right\| \leq c\left(\sum_{l=0}^{\infty} 2^{l s \beta}\left\|g_{l} \mid \dot{K}_{q}^{p}(\theta)\right\|^{\beta}\right)^{1 / \beta}
$$

and

$$
\left\|\sum_{l=0}^{\infty} g_{l}\left|\dot{K}_{q}^{p} F_{\beta}^{s}(\theta)\|\leq c\|\left(\sum_{l=0}^{\infty} 2^{l s \beta}\left|g_{l}\right|^{\beta}\right)^{1 / \beta}\right| \dot{K}_{q}^{p}(\theta)\right\|, \quad 1 \leq p, q<\infty .
$$


Before the proof of Proposition 1, we need some technical lemmas. The following assertion is the $\dot{K}_{q}^{p}(\theta)$-version of lemma by J. Franke [5].

Lemma 1. Let $\theta \in \mathcal{A}(\alpha, \delta), 1<p, q \leq \infty$ and $\gamma, \rho>1$. For any sequence $\left\{g_{l}\right\}_{l \in \mathbb{N}_{0}} \subset$ $\mathcal{S}^{\prime}\left(\mathbb{R}^{n}\right) \cap \dot{K}_{q}^{p}(\theta)$ with

$$
\operatorname{supp} \mathcal{F} g_{0} \subset\{\xi:|\xi| \leq \rho\} \quad \text { and } \quad \operatorname{supp} \mathcal{F} g_{l} \subset\left\{\xi: \gamma^{-1} 2^{l} \leq|\xi| \leq \gamma 2^{l}\right\},
$$

we have

$$
\left\|\Delta_{j} g_{l}\left|\dot{K}_{q}^{p}(\theta)\|\leq c\| g_{l}\right| \dot{K}_{q}^{p}(\theta)\right\| .
$$

The constant $c>0$ is independent of $j$ and $l$.

For the proof of this lemma, we can repeat arguments similar to the ones used in the proof of Lemma 3.3 in [2].

Lemma 2. Let $0<b<1$ and $0<q \leq \infty$. Let $\left\{\varepsilon_{j}\right\}_{j \in \mathbb{Z}}$ be a sequence of real positive numbers in $\ell_{q}$. Then there exists a constant $c>0$ depending only on $b$ and $q$ such that

$$
\left\|\left\{\sum_{j=-\infty}^{k} b^{(k-j)} \varepsilon_{j}\right\}_{k \in \mathbb{Z}}\left|\ell_{q}\|+\|\left\{\sum_{j=k}^{\infty} b^{(j-k)} \varepsilon_{j}\right\}_{k \in \mathbb{Z}}\right| \ell_{q}\right\| \leq c\left\|\left\{\varepsilon_{k}\right\}_{k \in \mathbb{Z}} \mid \ell_{q}\right\| .
$$

The proof of Lemma 2 is immediate by using Young's inequality in $\ell_{q}$.

Proof of Proposition 1. By similarity we prove only the Herz-type Besov case. We observe that there existe $H_{1}=\left[\log _{2} 2 \gamma\right]$ and $H_{2}=\left[\log _{2}((3 \gamma) / 2)\right]$ in $\mathbb{N}$ such that

$$
\Delta_{j} g_{l}=0 \quad \text { if } \quad l \geq j+H_{2} \quad \text { or } \quad l \leq j+H_{1} \text {. }
$$

Observe that

$$
\Delta_{j}\left(\sum_{l=0}^{\infty} g_{l}\right)=\sum_{l=j-H_{1}}^{j+H_{2}} \Delta_{j} g_{l}
$$

Therefore,

$$
\left\|\sum_{l=0}^{\infty} g_{l} \mid \dot{K}_{q}^{p} B_{\beta}^{s}(\theta)\right\| \leq\left(\sum_{j=0}^{\infty} 2^{j s \beta}\left(\sum_{l=j-H_{1}}^{j+H_{2}}\left\|\Delta_{j} g_{l} \mid \dot{K}_{q}^{p}(\theta)\right\|\right)^{\beta}\right)^{1 / \beta}
$$

Now, according to sign of $s$ and by Lemma 1, we separate the cases.

1 . The case $s>0$. We obtain

$\sum_{l=j-H_{1}}^{j+H_{2}} 2^{s j}\left\|\Delta_{j} g_{l} \mid \dot{K}_{q}^{p}(\theta)\right\| \leq c \sum_{l=j}^{\infty} 2^{s j} 2^{-s l}\left(2^{s l}\left\|\Delta_{j+H_{1}} g_{l} \mid \dot{K}_{q}^{p}(\theta)\right\|\right) \leq c 2^{s j} \sum_{l=j}^{\infty} 2^{-s l}\left(2^{s l}\left\|g_{l} \mid \dot{K}_{q}^{p}(\theta)\right\|\right)$.

2. The case $s<0$. Similarly

$\sum_{l=j-H_{1}}^{j+H_{2}} 2^{s j}\left\|\Delta_{j} g_{l} \mid \dot{K}_{q}^{p}(\theta)\right\| \leq c \sum_{l=0}^{j} 2^{s j} 2^{-s l}\left(2^{s l}\left\|\Delta_{j-H_{2}} g_{l} \mid \dot{K}_{q}^{p}(\theta)\right\|\right) \leq c 2^{j s} \sum_{l=0}^{j} 2^{-s j}\left(2^{s l}\left\|g_{l} \mid \dot{K}_{q}^{p}(\theta)\right\|\right)$.

3. If $s=0$, we immediately get

$\sum_{l=j-H_{1}}^{j+H_{2}}\left\|\Delta_{j} g_{l}\left|\dot{K}_{q}^{p}(\theta)\left\|\leq c \sum_{l=j-H_{1}}^{j+H_{2}} 1\right\| g_{l}\right| \dot{K}_{q}^{p}(\theta)\right\| \leq c\left(\sum_{l=j-H_{1}}^{j+H_{2}} 1\right)^{1 / \beta^{\prime}}\left(\sum_{l=j-H_{1}}^{j+H_{2}}\left(\left\|g_{l} \mid \dot{K}_{q}^{p}(\theta)\right\|\right)^{\beta}\right)^{1 / \beta}$.

Finally, we apply $\ell_{\beta}$-norm and Lemma 2 , and obtain

$$
\left\|\sum_{l=0}^{\infty} g_{l} \mid \dot{K}_{q}^{p} B_{\beta}^{s}(\theta)\right\| \leq c\left(\sum_{l=0}^{\infty} 2^{l s \beta}\left\|g_{l} \mid \dot{K}_{q}^{p}(\theta)\right\|^{\beta}\right)^{1 / \beta} \text {. }
$$




\section{Localization of Herz-type Besov spaces}

In this section, we present three results concerning the localization property of generalized Herz, Herz-type Besov and Herz-type Triebel-Lizorkin spaces on the $\ell_{r}$ spaces.

We first need the concept of a localization spaces. Let $E$ be a Banach space of distributions. We associate on the space $E$ the following hypothesis.

(1) Translation invariance: if $\tau_{k}$ denotes the operator given by $\tau_{k} f(t)=f(t-k)$, then $\tau_{k}$ is an isometry of $E$.

(2) Localization invariance: for all $f \in E$ and $\varphi \in \mathcal{D}\left(\mathbb{R}^{n}\right)$, we have that $\varphi \cdot f \in E$.

Let $\varphi \in \mathcal{D}\left(\mathbb{R}^{n}\right)$. The notion of localized is defined by $f_{x}=\tau_{x} \varphi \cdot f$, it follows immediately from the hypothesis (1) and (2) that the family $\left(f_{x}\right)_{x \in \mathbb{R}^{n}}$ is bounded in $E$. We consider the set $A$ as the class of all the functions $\varphi \in \mathcal{D}\left(\mathbb{R}^{n}\right)$ satisfying

$$
\operatorname{supp} \varphi \subset B(0, R) \text { with } R>\sqrt{n} \text {, }
$$

and

$$
\sum_{k \in \mathbb{Z}^{n}} \varphi(x-k)=1, \quad \forall x \in \mathbb{R}^{n} \backslash\{0\} .
$$

Definition 6. Let $E$ be a Banach space of distributions, $E$ is localizable in the $\ell_{r}$ norm, $1 \leq r \leq \infty$, if there exist $\varphi \in A$ and a constant $c \geq 1$ such that

$$
\frac{1}{c}\left\|f|E\|\leq\| f|(E)_{\ell_{r}}\right\|=\left(\sum_{k \in \mathbb{Z}^{n}}\left\|\tau_{k} \varphi \cdot f \mid E\right\|^{r}\right)^{1 / r} \leq c\|f \mid E\| .
$$

Remark 1. Let $f \in(E)_{\ell_{r}}$. If $g \in \mathcal{S}$ such that $g(x) \neq 0$ for $x \in \operatorname{supp} \varphi$. Then the following expression

$$
\left(\sum_{k \in \mathbb{Z}^{n}}\left\|\tau_{k} g \cdot f \mid E\right\|^{r}\right)^{1 / r}
$$

defines an equivalent norm in $(E)_{\ell_{r}}, c f$. [1, Proposition 5, p. 156].

The following result play a fundamental role in the proof of Theorem 3.

Lemma 3. Let $\theta \in \mathcal{A}(\alpha, \delta)$ and $1 \leq p, q \leq \infty$. There exists a constant $c>0$ such that the inequality

$$
\left\|\sum_{k \in \mathbb{Z}^{n}} f_{k} \mid \dot{K}_{q}^{p}(\theta)\right\| \leq c\left(\sum_{k \in \mathbb{Z}^{n}}\left\|f_{k} \mid \dot{K}_{q}^{p}(\theta)\right\|^{p}\right)^{1 / p}
$$

holds, for all $R>1$ and for all family $\left\{f_{k}\right\}_{k \in \mathbb{Z}^{n}}$ from $\mathcal{S}^{\prime}$ with supp $f_{k}$ contained in the ball $|x-k| \leq R$.

Proof. For any sequence $\left\{f_{k}\right\}_{k \in \mathbb{Z}^{n}}$, let us write $\sum_{k \in \mathbb{Z}^{n}} f_{k}$ as in (2), namely

$$
\sum_{k \in \mathbb{Z}^{n}} f_{k}=\sum_{k \in \mathbb{Z}^{n}} \varphi(x-k) f_{k}=\Lambda_{\varphi}\left(\left\{f_{k}\right\}_{k \in \mathbb{Z}^{n}}\right)
$$

where $\varphi \in \mathcal{D}\left(\mathbb{R}^{n}\right)$ is chosen such that $\varphi=1$ on the ball $|x| \leq R$. We want to show that $\Lambda_{\varphi}$ is bounded operator from $\ell_{1}\left(\dot{K}_{1}^{p}(\theta)\right)$ to $\dot{K}_{1}^{p}(\theta)$ and from $\ell_{\infty}\left(\dot{K}_{\infty}^{p}(\theta)\right)$ to $\dot{K}_{\infty}^{p}(\theta)$. 
Consider $\left\|\Lambda_{\varphi}\left(\left\{f_{k}\right\}_{k \in \mathbb{Z}^{n}}\right) \mid \dot{K}_{1}^{p}(\theta)\right\|$ first. By Minkowski's inequality, Hölder's inequality and since $p \geq 1$, we have

$$
\begin{aligned}
\left\|\Lambda_{\varphi}\left(\left\{f_{k}\right\}_{k \in \mathbb{Z}^{n}}\right) \mid \dot{K}_{1}^{p}(\theta)\right\| & =\left(\sum_{l=-\infty}^{\infty} \theta^{p}(l)\left\|\sum_{k \in \mathbb{Z}^{n}} \varphi(\cdot-k) f_{k} \cdot \chi_{l} \mid L^{1}\right\|^{p}\right)^{1 / p} \\
& \leq\|\| \theta(l) \varphi(\cdot-k) f_{k} \cdot \chi_{l}\left|L^{1}\left\|\mid \ell_{p}\left(\ell_{1}\right)\right\|\right. \\
& \leq\left\|\varphi \left|L ^ { \infty } \| \| \left\|\theta ( l ) f _ { k } \cdot \chi _ { l } \left|L^{1}\left\|\mid \ell_{p}\left(\ell_{1}\right)\right\|\right.\right.\right.\right. \\
& \leq\|\| \theta(l) f_{k} \cdot \chi_{l}\left|L^{1}\left\|\mid \ell_{1}\left(\ell_{p}\right)\right\|\right. \\
& \leq \sum_{k \in \mathbb{Z}^{n}}\left\|f _ { k } \left|\dot { K } _ { 1 } ^ { p } ( \theta ) \| = \| \left\|( f _ { k } ) _ { k \in \mathbb { Z } ^ { n } } \left|\dot{K}_{1}^{p}(\theta)\left\|\mid \ell_{1}\right\| .\right.\right.\right.\right.
\end{aligned}
$$

We now consider $\left\|\Lambda_{\varphi}\left(\left\{f_{k}\right\}_{k \in \mathbb{Z}^{n}}\right) \mid \dot{K}_{\infty}^{p}(\theta)\right\|$. By Hölder's inequality, we have

$$
\begin{aligned}
\left\|\Lambda_{\varphi}\left(\left\{f_{k}\right\}_{k \in \mathbb{Z}^{n}}\right) \mid \dot{K}_{\infty}^{p}(\theta)\right\| & =\left(\sum_{l=-\infty}^{\infty} \theta^{p}(l)\left\|\sum_{k \in \mathbb{Z}^{n}} \varphi(\cdot-k) f_{k} \cdot \chi_{l} \mid L^{\infty}\right\|^{p}\right)^{1 / p} \\
& \leq\left(\sum_{l=-\infty}^{\infty} \theta^{p}(l)\left\|\sup _{k \in \mathbb{Z}^{n}} f_{k} \cdot \chi_{l}\left|L^{\infty}\left\|^{p}\right\| \sum_{k \in \mathbb{Z}^{n}} \varphi(\cdot-k)\right| L^{\infty}\right\|^{p}\right)^{1 / p} \\
& \leq\|\|\left(f_{k}\right)_{k \in \mathbb{Z}^{n}}\left|\dot{K}_{\infty}^{p}(\theta)\left\|\mid \ell_{\infty}\right\| .\right.
\end{aligned}
$$

Since $\frac{1}{p} \in(0,1)$, by the complex interpolation theory established in [3, Theorem 4.1] and $[12$, p. 121/4]), we have

$$
\left[\ell_{1}\left(\dot{K}_{1}^{1}(\theta)\right), \ell_{\infty}\left(\dot{K}_{\infty}^{\infty}(\theta)\right)\right]_{\frac{1}{p}}=\ell_{p}\left(\left[\dot{K}_{1}^{1}(\theta), \dot{K}_{\infty}^{\infty}(\theta)\right]_{\frac{1}{p}}\right)=\ell_{p}\left(\dot{K}_{p}^{p}(\theta)\right) .
$$

This finishes the proof of the lemma.

The following result gives the localization property of generalized Herz spaces on the $\ell_{r}$ spaces.

Theorem 1. Let $\theta \in \mathcal{A}(\alpha, \delta), 1<p, q \leq \infty$. Then

(i) $\dot{K}_{q}^{p}(\theta) \hookrightarrow\left(\dot{K}_{q}^{p}(\theta)\right)_{\ell_{r}}$ for $r \geq \max (p, q)$ ，

(ii) $\left(\dot{K}_{q}^{p}(\theta)\right)_{\ell_{r}} \hookrightarrow \dot{K}_{q}^{p}(\theta)$ for $r \leq \min (p, q)$.

In particular, $\dot{K}_{q}^{q}(\theta)$ space is localizable in the $\ell_{q}$-norm.

Proof. (i) We must show that

$$
\left\|f\left|\left(\dot{K}_{q}^{p}(\theta)\right)_{\ell_{r}}\|\leq c\| f\right| \dot{K}_{q}^{p}(\theta)\right\|
$$

for all $f \in \dot{K}_{q}^{p}(\theta)$. We have

$$
\left\|\tau_{k} \varphi \cdot f\left|\dot{K}_{q}^{p}(\theta)\|=\| \sum_{l=-\infty}^{\infty} \tau_{k} \varphi \cdot f \cdot \chi_{l}\right| \dot{K}_{q}^{p}(\theta)\right\|=\left(\sum_{k=-\infty}^{\infty} \theta^{p}(k)\left\|\sum_{l=-\infty}^{\infty} \tau_{k} \varphi \cdot f \cdot \chi_{l} \cdot \chi_{k} \mid L^{q}\right\|^{p}\right)^{1 / p} .
$$


We observe that $\chi_{l} \cdot \chi_{k} \neq 0$, when $|l-k|<1$, which means $l=k$. We have

$$
\left\|\tau_{k} \varphi \cdot f \mid \dot{K}_{q}^{p}(\theta)\right\| \leq\left(\sum_{l=-\infty}^{\infty} \theta^{p}(l)\left\|\tau_{k} \varphi \cdot f \cdot \chi_{l} \mid L^{q}\right\|^{p}\right)^{1 / p}
$$

this implies

$$
\left\|f \mid\left(\dot{K}_{q}^{p}(\theta)\right)_{\ell_{r}}\right\|=\left(\sum_{k \in \mathbb{Z}^{n}}\left\|\tau_{k} \varphi \cdot f \mid \dot{K}_{q}^{p}(\theta)\right\|^{r}\right)^{1 / r} \leq\left(\sum_{k \in \mathbb{Z}^{n}}\left(\sum_{l=-\infty}^{\infty} \theta^{p}(l)\left\|\tau_{k} \varphi \cdot f \cdot \chi_{l} \mid L^{q}\right\|^{p}\right)^{r / p}\right)^{1 / r} .
$$

Since $r \geq \max (p, q)$ implies $r \geq p$, from Minkowski inequality we have

$$
\left\|f \mid\left(\dot{K}_{q}^{p}(\theta)\right)_{\ell_{r}}\right\| \leq\left(\sum_{l=-\infty}^{+\infty} \theta^{p}(l)\left(\sum_{k \in \mathbb{Z}^{n}}\left\|\tau_{k} \varphi \cdot f \cdot \chi_{l} \mid L^{q}\right\|^{r}\right)^{p / r}\right)^{1 / p} .
$$

Since $r \geq \max (p, q) \geq q$, by the embedding $\ell_{q} \hookrightarrow \ell_{r}$ and the localization of $L^{q}$ in $\ell^{q}$ spaces we obtain

$$
\left(\sum_{k \in \mathbb{Z}^{n}}\left\|\tau_{k} \varphi \cdot f \cdot \chi_{l} \mid L^{q}\right\|^{r}\right)^{1 / r} \leq\left(\sum_{k \in \mathbb{Z}^{n}}\left\|\tau_{k} \varphi \cdot f \cdot \chi_{l} \mid L^{q}\right\|^{q}\right)^{1 / q} \leq c\left\|f \cdot \chi_{l} \mid L^{q}\right\|
$$

the right hand side inequality of (3) is bounded by

$$
\left\|f\left|\left(\dot{K}_{q}^{p}(\theta)\right)_{\ell_{r}}\left\|\leq c\left(\sum_{l=-\infty}^{\infty} \theta^{p}(l)\left\|f \cdot \chi_{l} \mid L^{q}\right\|^{p}\right)^{1 / p}=c\right\| f\right| \dot{K}_{q}^{p}(\theta)\right\| .
$$

(ii) By the localization of $L^{q}$ spaces in the $\ell_{q}$ norm (with $r \leq \min (p, q) \leq q$ ), we have

$$
\begin{aligned}
\left\|f \cdot \chi_{l}\left|L^{q}\|=\| \sum_{k \in \mathbb{Z}^{n}} \tau_{k} \varphi \cdot f \cdot \chi_{l}\right| L^{q}\right\| & \leq c\left(\sum_{k \in \mathbb{Z}^{n}}\left\|\tau_{k} \varphi \cdot f \cdot \chi_{l} \mid L^{q}\right\|^{q}\right)^{1 / q} \\
& \leq c\left(\sum_{k \in \mathbb{Z}^{n}}\left\|\tau_{k} \varphi \cdot f \cdot \chi_{l} \mid L^{q}\right\|^{r}\right)^{1 / r}
\end{aligned}
$$

this implies that

$$
\left\|f \mid \dot{K}_{q}^{p}(\theta)\right\|=\left(\sum_{l=-\infty}^{\infty} \theta^{p}(l)\left\|f \cdot \chi_{l} \mid L^{q}\right\|^{p}\right)^{1 / p} \leq c\left(\sum_{l=-\infty}^{+\infty} \theta^{p}(l)\left(\sum_{k \in \mathbb{Z}^{n}}\left\|\tau_{k} \varphi \cdot f \cdot \chi_{l} \mid L^{q}\right\|^{r}\right)^{p / r}\right)^{1 / p} .
$$

Since $r \leq \min (p, q)$, it holds that $r \leq p$, then from Minkowski inequality we have

$$
\left\|f\left|\dot{K}_{q}^{p}(\theta)\left\|\leq c\left(\sum_{k \in \mathbb{Z}^{n}}\left(\sum_{\ell=-\infty}^{\infty} 2^{l \alpha p}\left\|\tau_{k} \varphi \cdot f \cdot \chi_{l} \mid L^{q}\right\|^{p}\right)^{r / p}\right)^{1 / r}=\right\| f\right|\left(\dot{K}_{q}^{p}(\theta)\right)_{\ell_{r}}\right\| .
$$

This finishes the proof of the theorem.

Remark 2. We would like to mention if $\theta \in \mathcal{A}(0,0)$ and $p=q$, then the statements corresponding to Theorem 1 present the localization of Lebesgue spaces $L^{q}$ on the $\ell_{q}$ spaces.

Motivated by $[1,4,13]$, we give the localization property of generalized Herz-type Besov and Herz-type Triebel-Lizorkin spaces on the $\ell_{r}$ spaces. 
Theorem 2. Let $\theta \in \mathcal{A}(\alpha, \delta), s \in \mathbb{R}, 1 \leq p, q, \beta \leq \infty$ such that $-n / q<\alpha, \delta<n(1-1 / q)$. Then

(i) $\dot{K}_{q}^{p} B_{\beta}^{s}(\theta) \hookrightarrow\left(\dot{K}_{q}^{p} B_{\beta}^{s}(\theta)\right)_{\ell_{r}}$ for $r \geq \max (p, q, \beta)$,

(ii) $\left(\dot{K}_{q}^{p} B_{\beta}^{s}(\theta)\right)_{\ell_{r}} \hookrightarrow \dot{K}_{q}^{p} B_{\beta}^{s}(\theta)$ for $r \leq \min (p, q, \beta)$.

In particular, $\dot{K}_{q}^{q} B_{q}^{s}(\theta)$ space is localizable in the $\ell_{q}$-norm.

Proof. Our proofs use partially some techniques already used in [4], where Besov spaces case was studied.

First, we prove (i). We must show that

$$
\left\|f\left|\left(\dot{K}_{q}^{p} B_{\beta}^{s}(\theta)\right)_{\ell_{r}}\|\leq c\| f\right| \dot{K}_{q}^{p} B_{\beta}^{s}(\theta)\right\|
$$

for all $f \in \dot{K}_{q}^{p} B_{\beta}^{s}(\theta)$. By Proposition 1 , we have

$$
\left\|\tau_{k} \varphi \cdot f\left|\dot{K}_{q}^{p} B_{\beta}^{s}(\theta)\|=\| \sum_{j=0}^{\infty} \tau_{k} \varphi \cdot \Delta_{j} f\right| \dot{K}_{q}^{p} B_{\beta}^{s}(\theta)\right\| \leq\left(\sum_{j=0}^{\infty} 2^{j s \beta}\left\|\tau_{k} \varphi \cdot \Delta_{j} f \mid \dot{K}_{q}^{p}(\theta)\right\|^{\beta}\right)^{1 / \beta},
$$

this implies that,

$\left\|f \mid\left(\dot{K}_{q}^{p} B_{\beta}^{s}(\theta)\right)_{\ell_{r}}\right\|=\left(\sum_{k \in \mathbb{Z}^{n}}\left\|\tau_{k} \varphi \cdot f \mid \dot{K}_{q}^{p} B_{\beta}^{s}(\theta)\right\|^{r}\right)^{1 / r} \leq\left(\sum_{k \in \mathbb{Z}^{n}}\left(\sum_{j=0}^{\infty} 2^{j s \beta}\left\|\tau_{k} \varphi \cdot \Delta_{j} f \mid \dot{K}_{q}^{p}(\theta)\right\|^{\beta}\right)^{r / \beta}\right)^{1 / r}$.

Since $r \geq \max (p, q, \beta)$ implies $r \geq \beta$, from Minkowski inequality we have

$$
\left\|f \mid\left(\dot{K}_{q}^{p} B_{\beta}^{s}(\theta)\right)_{\ell_{r}}\right\| \leq\left(\sum_{j=0}^{\infty} 2^{j s \beta}\left(\sum_{k \in \mathbb{Z}^{n}}\left\|\tau_{k} \varphi \cdot \Delta_{j} f \mid \dot{K}_{q}^{p}(\theta)\right\|^{r}\right)^{\beta / r}\right)^{1 / \beta}
$$

Since $\dot{K}_{q}^{p}(\theta) \hookrightarrow\left(\dot{K}_{q}^{p}(\theta)\right)_{\ell_{r}}$, i.e.

$$
\left(\sum_{k \in \mathbb{Z}^{n}}\left\|\tau_{k} \varphi \cdot \Delta_{j} f \mid \dot{K}_{q}^{p}(\theta)\right\|^{r}\right)^{1 / r} \leq c\left\|\Delta_{j} f \mid \dot{K}_{q}^{p}(\theta)\right\|
$$

the right hand side inequality of (4) is bounded by

$$
\left\|f\left|\left(\dot{K}_{q}^{p} B_{\beta}^{s}(\theta)\right)_{\ell_{r}}\left\|\leq\left(\sum_{j=0}^{\infty} 2^{j s \beta}\left\|\Delta_{j} f \mid \dot{K}_{q}^{p}(\theta)\right\|^{\beta}\right)^{1 / \beta}=\right\| f\right| \dot{K}_{q}^{p} B_{\beta}^{s}(\theta)\right\| .
$$

(ii) By the localization of Herz spaces in the $\ell_{r}$-norm (with $r \leq \min (p, q)$, see Theorem 2 (ii)), we have

$$
\left\|\Delta_{j} f\left|\dot{K}_{q}^{p}(\theta)\|=\| \sum_{k \in \mathbb{Z}^{n}} \tau_{k} \varphi \cdot \Delta_{j} f\right| \dot{K}_{q}^{p}(\theta)\right\| \leq c\left(\sum_{k \in \mathbb{Z}^{n}}\left\|\tau_{k} \varphi \cdot \Delta_{j} f \mid \dot{K}_{q}^{p}(\theta)\right\|^{r}\right)^{1 / r},
$$

this implies that

$$
\left\|f \mid \dot{K}_{q}^{p} B_{\beta}^{s}(\theta)\right\|=\left(\sum_{j=0}^{\infty} 2^{j s \beta}\left\|\Delta_{j} f \mid \dot{K}_{q}^{p}(\theta)\right\|^{\beta}\right)^{1 / \beta} \leq c\left(\sum_{j=0}^{\infty} 2^{j s \beta}\left(\sum_{k \in \mathbb{Z}^{n}}\left\|\tau_{k} \varphi \cdot \Delta_{j} f \mid \dot{K}_{q}^{p}(\theta)\right\|^{r}\right)^{\beta / r}\right)^{1 / \beta} .
$$

Since $r \leq \min (p, q, \beta)$, it holds that $r \leq q$, then from Minkowski inequality we have

$$
\begin{aligned}
\left\|f \mid \dot{K}_{q}^{p} B_{\beta}^{s}(\theta)\right\| & =\left(\sum_{j=0}^{\infty} 2^{j s \beta}\left(\sum_{k \in \mathbb{Z}^{n}}\left\|\tau_{k} \varphi \cdot \Delta_{j} f \mid \dot{K}_{q}^{p}(\theta)\right\|^{r}\right)^{\beta / r}\right)^{1 / \beta} \\
& \leq\left(\sum_{k \in \mathbb{Z}^{n}}\left(\sum_{j=0}^{\infty} 2^{j s \beta}\left\|\tau_{k} \varphi \cdot \Delta_{j} f \mid \dot{K}_{q}^{p}(\theta)\right\|^{\beta}\right)^{r / \beta}\right)^{1 / r}=\left\|f \mid\left(\dot{K}_{q}^{p} B_{\beta}^{s}(\theta)\right)_{\ell_{r}}\right\| .
\end{aligned}
$$

This finishes the proof of the theorem. 
Remark 3. We would like to mention if $\theta \in \mathcal{A}(0,0)$ and $p=q$, then the statements corresponding to Theorem 2 can be found in Theorem 2 of [4].

Theorem 3. Let $\theta \in \mathcal{A}(\alpha, \delta), s \in \mathbb{R}, 1 \leq p, q, \beta \leq \infty$ such that $-n / q<\alpha, \delta<n(1-1 / q)$. Then

$$
\dot{K}_{q}^{p} F_{\beta}^{s}(\theta)=\left(\dot{K}_{q}^{p} F_{\beta}^{s}(\theta)\right)_{\ell_{q}} .
$$

Proof. First, we prove $\left(\dot{K}_{q}^{p} F_{\beta}^{s}(\theta)\right)_{\ell_{q}} \hookrightarrow \dot{K}_{q}^{p} F_{\beta}^{s}(\theta)$. We must show that

$$
\left\|f\left|\dot{K}_{q}^{p} F_{\beta}^{s}(\theta)\|\leq c\| f\right|\left(\dot{K}_{q}^{p} F_{\beta}^{s}(\theta)\right)_{\ell_{q}}\right\|
$$

for all $f \in\left(\dot{K}_{q}^{p} F_{\beta}^{s}(\theta)\right)_{\ell_{q}}$. We have

$$
\begin{aligned}
\left\|f\left|\dot{K}_{q}^{p} F_{\beta}^{s}(\theta)\|=\|\left(\sum_{j=0}^{\infty} 2^{j s \beta}\left|\Delta_{j} f\right|^{\beta}\right)^{1 / \beta}\right| \dot{K}_{q}^{p}(\theta)\right\| & \leq\left\|\left(\sum_{j=0}^{\infty}\left|\sum_{k \in \mathbb{Z}^{n}} 2^{j s} \tau_{k} \varphi \cdot \Delta_{j} f\right|^{\beta}\right)^{1 / \beta} \mid \dot{K}_{q}^{p}(\theta)\right\| \\
& \leq\|\| 2^{j s} \tau_{k} \varphi \cdot \Delta_{j} f\left|\ell_{\beta}\left(\ell_{1}\right)\left\|\mid \dot{K}_{q}^{p}(\theta)\right\| .\right.
\end{aligned}
$$

Since $\beta \geq 1$, by Minkowski inequality, Lemma 3 and Remark 1, we have

$$
\begin{aligned}
\left\|f \mid \dot{K}_{q}^{p} F_{\beta}^{s}(\theta)\right\| & \leq c\|\| 2^{j s} \tau_{k} \varphi \cdot \Delta_{j} f\left|\ell_{1}\left(\ell_{\beta}\right)\left\|\mid \dot{K}_{q}^{p}(\theta)\right\|\right. \\
& =c\left\|\sum_{k \in \mathbb{Z}^{n}}\left(\sum_{j=0}^{\infty}\left|2^{j s} \tau_{k} \varphi \cdot \Delta_{j} f\right|^{\beta}\right)^{1 / \beta} \mid \dot{K}_{q}^{p}(\theta)\right\| \\
& \leq c \sum_{k \in \mathbb{Z}^{n}}\left\|\tau_{k} \varphi \cdot \Delta_{j} f \mid \dot{K}_{q}^{p} F_{\beta}^{s}(\theta)\right\| \leq c\left(\sum_{k \in \mathbb{Z}^{n}}\left\|\tau_{k} \varphi \cdot f \mid \dot{K}_{q}^{p} F_{\beta}^{s}(\theta)\right\|^{q}\right)^{1 / q} \\
& \leq c\left\|f \mid\left(\dot{K}_{q}^{p} F_{\beta}^{s}(\theta)\right)_{\ell_{q}}\right\| .
\end{aligned}
$$

Second, we prove $\dot{K}_{q}^{p} F_{\beta}^{s}(\theta) \hookrightarrow\left(\dot{K}_{q}^{p} F_{\beta}^{s}(\theta)\right)_{\ell_{q}}$. We must show that

$$
\left\|f\left|\left(\dot{K}_{q}^{p} F_{\beta}^{s}(\theta)\right)_{\ell_{q}}\|\leq c\| f\right| \dot{K}_{q}^{p} F_{\beta}^{s}(\theta)\right\|
$$

for all $f \in \dot{K}_{q}^{p} F_{\beta}^{s}(\theta)$. We have

$$
\left\|f \mid\left(\dot{K}_{q}^{p} F_{\beta}^{s}(\theta)\right)_{\ell_{q}}\right\|=\left(\sum_{k \in \mathbb{Z}^{n}}\left\|\tau_{k} \varphi \cdot f \mid \dot{K}_{q}^{p} F_{\beta}^{s}(\theta)\right\|^{q}\right)^{1 / q}=\left(\sum_{k \in \mathbb{Z}^{n}}\left\|\sum_{j=0}^{+\infty} \tau_{k} \varphi \cdot \Delta_{j} f \mid \dot{K}_{q}^{p} F_{\beta}^{s}(\theta)\right\|^{q}\right)^{1 / q} .
$$

By Proposition 1, we obtain

$$
\left\|f \mid\left(\dot{K}_{q}^{p} F_{\beta}^{s}(\theta)\right)_{\ell_{q}}\right\| \leq c\left(\sum_{k \in \mathbb{Z}^{n}}\left\|\left(\sum_{j=0}^{\infty}\left|2^{j s} \tau_{k} \varphi \cdot \Delta_{j} f\right|^{\beta}\right)^{1 / \beta} \mid \dot{K}_{q}^{p}(\theta)\right\|^{q}\right)^{1 / q} .
$$

By Theorem 2 (i) with $r=q$, the right hand side inequality of the last inequality is bounded by

$$
c\left\|\left(\sum_{j=0}^{\infty}\left|2^{j s} \tau_{k} \varphi \cdot \Delta_{j} f\right|^{\beta}\right)^{1 / \beta}\left|\dot{K}_{q}^{p}(\theta)\|=c\| f\right| \dot{K}_{q}^{p} F_{\beta}^{s}(\theta)\right\| .
$$

This finishes the proof of the Theorem. 
Remark 4. We would like to mention if $\theta \in \mathcal{A}(0,0)$ and $p=q$, then the statements corresponding to Theorem 3 can be found in Theorem 3 of [4].

\section{Acknowledgement}

This work is supported by the General Direction of Scientific Research and Technological Development. PRFU Projects №C00L03UN280120180007 and №C00L03UN280120180002.

\section{References}

[1] Bourdaud G. Localisations des espaces de Besov. Studia Math. 1988, 90 (2), 153-163. (in French)

[2] Djeriou A., Drihem D. On the Continuity of Pseudo-Differential Operators on Multiplier Spaces Associated to Herztype Triebel-Lizorkin Spaces. Mediterr. J. Math. 2019, 16 153. doi:10.1007/s00009-019-1418-7

[3] Drihem D. Complex interpolation of Herz-type Triebel-Lizorkin spaces. Math. Nachr. 2018, 291 (13), 2008-2023. doi:10.1002/mana.201700266

[4] Ferahtia N., Allaoui S. A generalization of a localization property of Besov spaces. Carpathian Math. Publ. 2018, 10 (1), 71-78. doi:10.15330/cmp.10.1.71-78

[5] Franke J. On the spaces of triebel-lizorkin type: Pointwise multipliers and spaces on domains. Math. Nachr. 1986, 125 (1), 29-68. doi:10.1002/mana.19861250104

[6] Hernández E., Weiss G., Yang D. The $\varphi$-transform and wavelet characterizations of Herz-type spaces. Collect. Math. 1996, 47 (3), 285-320.

[7] Hernández E., Yang D. Interpolation of Herz-type Hardy spaces. Illinois J. Math. 1998, 42 (4), 564-581. doi:10.1215/ijm/1255985461

[8] Komori Y., Matsuoka K. Boundedness of several operators on weighted Herz spaces. J. Funct. Spaces 2009, 7 (1), 1-12. doi.org/10.1155/2009/739134

[9] Lu S.Z. The local versions of $H^{p}\left(\mathbb{R}^{n}\right)$ spaces at the origin. Studia Math. 1995, 116, 103-131. doi:10.4064/sm-1162-103-131

[10] Lu S., Yang D. The decomposition of weighted Herz spaces on $\mathbb{R}^{n}$ and its applications. Science in China (Ser.A). $1995,38,147-158$.

[11] Sickel W., Smirnov I. Localization properties of Besov spaces and of its associated multiplier spaces. Jenaer Schriftenzur Mathematik und Informatik 1999. Math/Inf/99/21.

[12] Triebel H. Interpolation Theory, Function Spaces, Differential Operators. North-Holland, 1978.

[13] Triebel H. A localization property for $B_{p, q}^{s}$ and $F_{p, q}^{s}$ spaces. Studia Math. 1994, 109, 183-195. doi:10.4064/sm-1092-183-195

[14] Triebel H. Theory of function spaces I. Birkhäuser, Basel, 1983.

[15] Triebel H. Theory of function spaces II. Birkhäuser, Basel, 1992.

[16] Triebel H. Theory of function spaces III. Birkhäuser Basel, 2006.

[17] Xu J., Yang D. Herz-type Triebel-Lizorkin Spaces, I. Acta Math. Sin. (Engl. Ser.) 2005, 21 (3), 643-654. doi:10.1007/s10114-004-0424-1

[18] Xu J. Some properties on Herz-type Besov spaces. J. Hunan Univ. (Nat. Sci) 2003, 30 (5), 1-4. (in Chinese) 
[19] Xu J. Equivalent norms of Herz-type Besov and Triebel-Lizorkin spaces. J. Funct. Spaces 2005, 3, 17-31. doi: $10.1155 / 2005 / 149703$

[20] Youssefi A. Localisation des espaces de Lizorkin-Triebel homogènes. Math. Nechr. 1990, 147, 107-121. doi: 10.1002/mana.19901470113

Received 05.04.2020

Revised 18.10.2020

Ажеріу А., Гераїз Р. Аеякі результати стосовно властивості локалізаиї узагальнених просторів Гериа, просторів Бесова типу Гериа і просторів Трібеля-Аізоркіна типу Гериа // Карпатські матем. публ. - 2021. - Т.13, №1. - С. 217-228.

У цій статті, використовуючи узагальнені функційні простори типу Герца $\dot{K}_{q}^{p}(\theta)$, що були введені Й. Коморі та К. Мацуока у 2009 році, ми визначаємо простори Бєсова типу Герца $\dot{K}_{q}^{p} B_{\beta}^{s}(\theta)$ і простори Трібеля- $\Lambda$ ізоркіна типу Герца $\dot{K}_{q}^{p} F_{\beta}^{s}(\theta)$, які узагальнюють простори Бєсова і простори Трібеля- $\Lambda$ ізоркіна в однорідному випадку, де $\theta=\{\theta(k)\}_{k \in \mathbb{Z}}$ - така послідовність невід'ємних чисел, що

$$
C^{-1} 2^{\delta(k-j)} \leq \frac{\theta(k)}{\theta(j)} \leq C 2^{\alpha(k-j)}, \quad k>j,
$$

для деякого $C \geq 1$ ( $\alpha$ і $\delta-$ дійсні числа).

При зазначених вище умовах на $\theta$ ми доводимо, що $\dot{K}_{q}^{p}(\theta)$ і $\dot{K}_{q}^{p} B_{\beta}^{s}(\theta) \in$ локалізовні у $\ell_{q}$-нормі при $p=q, \dot{K}_{q}^{p} F_{\beta}^{s}(\theta) \in$ локалізовні у $\ell_{q}$-нормі, тобто існує $\varphi \in \mathcal{D}\left(\mathbb{R}^{n}\right)$, що задовольняє $\sum_{k \in \mathbb{Z}^{n}} \varphi(x-k)=1$ для довільного $x \in \mathbb{R}^{n}$ так, шо

$$
\|f \mid E\| \approx\left(\sum_{k \in \mathbb{Z}^{n}}\|\varphi(\cdot-k) \cdot f \mid E\|^{q}\right)^{1 / q} .
$$

Вказані результати покрашують та узагальнюють відповідні відомі результати для деяких функційних просторів.

Ключові слова $і$ фрази: узагальнений простір Герца, простір Бєсова типу Герца, простір Трібеля-Аізоркіна типу Герца, властивість локалізаціі. 